\title{
LA GLOBALIZACIÓN Y LA SALUD EN LOS GRUPOS MINORITARIOS
}

\author{
Sergio Romeo LÓPEZ ALONSO \\ C/ Río Guadalquivir, 8 \\ Mijas-Costa CP: 29649 \\ Málaga \\ Teléfono: 952476890 - 656326107 \\ E-mail: sergiolopezalonso@yahoo.com
}

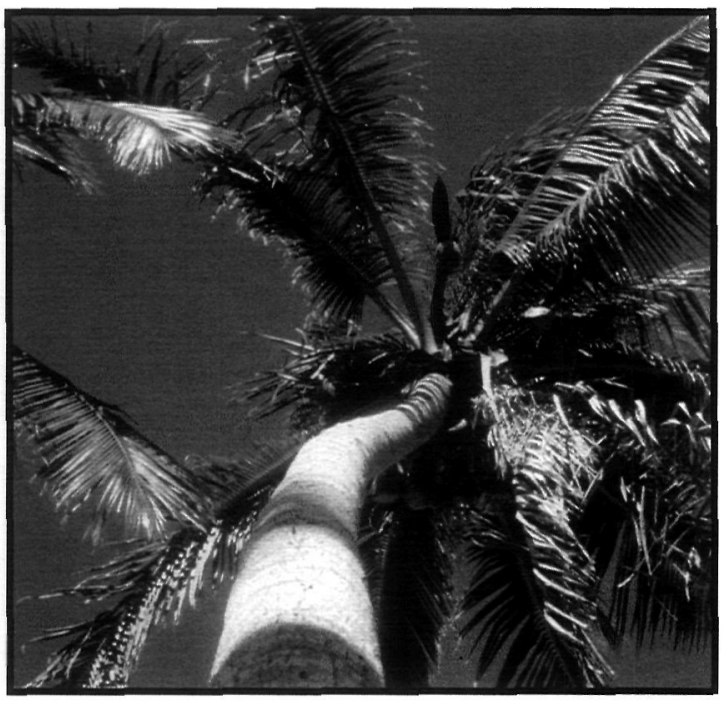

GLOBALIZATION AND HEALTH IN MINORITY GROUPS

7 he following article goes through the history of cultures, catching a glimpse of how they 1 merge and confront their identities by the unstoppable upcoming globalization. Likewise, the trends within each ethnic group in a multicultural society can be appreciated. In this context, a reference is made to the benefits and backdraws of emigration for the country of origin and for the destination country, where stress is made on the perceived discrepancies inside the realm of health. Furthermore, various philosophic ideologies and nursing theories are described, from which to cope effectively with racism and inequality occurred in health services.

\section{RESUMEN}

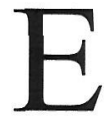
l siguiente artículo presenta un recorrido por la historia de las culturas, dejando entrever como se van confundiendo y confrontando sus identidades ante la irrefrenable entrada de la globalización. Asimismo, se pueden apreciar las tendencias que van tomando los distintos grupos étnicos dentro de una sociedad multicultural. En este contexto, se hace referencia a los beneficios e inconvenientes de la emigración tanto en el país de procedencia como en el de destino, donde se hace hincapié sobre las divergencias advertidas dentro del marco de la salud. Además, describe varias ideologías filosóficas y teorías enfermeras desde las que afrontar de forma efectiva el racismo y las desigualdades acaecidas en los servicios de salud.

\section{ETNOHISTORIA, VIEJAS Y NUEVAS IDENTIDADES}

De acuerdo con Leininger (1995), la etnohistoria se refiere a "aquellos hechos, eventos, situaciones y experiencias pasadas de individuos, grupos, culturas e instituciones que están centrados principalmente en las personas (etno-) y que describen, explican e interpretan los estilos de vida humanos en un contexto cultural particular a lo largo de periodos cortos o largos de tiempo"

Sin duda, la historia de cualquier ser humano ha estado siempre circundada por la cultura, y todos los individuos crecen bajo unas determinadas condiciones culturales. Este proceso de culturización es el que hace que los individuos sientan que controlan sus vidas (Segall, 1990), y también crean que les dan un significado individualizado. Pero este proceso puede ser modificado por la 
influencia de una cultura nueva y dominante, lo que se llama aculturación. Se han identificado dos niveles de aculturación (Foss, 1996): uno a nivel de la población, cuando los cambios se dan en la estructura social, económica y política; y otro a nivel individual, cuando se dan en la identidad, valores y comportamientos.

Hoy en día, el fenómeno de la emigración está aumentando el número de personas que sufren este proceso de aculturación $y$, lamentablemente, la aculturación también trae consigo problemas psicológicos y sociales. Diversos autores de teorías de Enfermería han creado algunas guías de valoración, con el fin de medir la consistencia cultural heredada, definida ésta como "el grado en el que el estilo de vida de alguien refleja su cultura tribal respectiva" (Spector, 1990). Con el uso de esta guía, los enfermeros podrán mitigar los problemas que pueden afectar a la salud, y ayudar a los inmigrantes durante este proceso, posiblemente, crítico.

La aculturación es una situación inevitable. Diferentes autores, como Anthias (1988) y Spector (1991), destacaron que el proceso para lograr la total aculturación conlleva el paso de tres generaciones. En otro sentido, Foss (1996) identificó tres categorías de incorporaciones frente a una nueva cultura: la marginalización; la integridad cultural, como el uso de diferentes identidades étnicas que forman parte de la auto-imagen y personalidad; y la asimilación social, como la pérdida de una identidad étnica. Aunque esta última puede ser argumentada, ya que todos los individuos, cuando se enfrentan ante una cultura nueva, tienden a asimilar aquellas partes que consideran útiles para su integridad e identidad. Difícilmente, alguiien entrará de lleno en una nueva cultura olvidando todos y cada uno de sus aspectos culturales anteriores, pues algunos de ellos son subconscientes. En nuestro presente, debido al aumento de la inmigración, las personas conviven dentro de una sociedad multicultural. Esta situación aporta nuevos útiles, percepciones, conocimientos y reflexiones, y consecuentemente la oportunidad de elegir y ser un poco más libre.

\section{EL FENÓMENO DE LA GLOBALIZACIÓN}

El desarrollo del mundo conlleva continuos cambios en la política, economía, cultura y en otras dimensiones. Todos estos aspectos se manifiestan por el aumento en la circulación de personas, bienes e información, y este desarrollo del mundo lleva a la globalización. Así, la globalización se describe como el proceso donde el aumento de interconexiones se da lugar en la cultura, economía y vida social (Barnarad, 1996).

Muchos autores han estudiado este fenómeno, y puntos de vista muy diferentes han salido a la luz. Desde el punto de vista positivo, se han identificado la conciencia y el reconocimiento de retos comunes, tales como la salud pública, la educación, la conservación del medio ambiente, el intercambio de información, los derechos humanos y el sistema legal. Como negativos, se han subrayado la explotación económica y el imperialismo cultural, ambos debido a un proceso desigual que sigue a la polarización entre los países beneficiados por este proceso y los que son manipulados y extorsionados por los primeros (Human Development Report, 1999).

Como consecuencia de ello, se ha llegado a la conclusión de que el desarrollo del mundo no está bien orientado. La globalización puede traer tanto polarización como equilibrio, por tanto, se hace necesaria una intervención más humana y consciente. En este proceso, una política racional y un buen uso de la tecnología se tornan esenciales. La equidad en salud es un pilar básico con el fin de lograr de forma efectiva el desarrollo uniforme en otras áreas fundamentales, como la economía.

La llegada de personas a países extranjeros conlleva que los inmigrantes perciban un sentimiento de pérdida de identidad, debido a que experimentan una "dislocación" en el tiempo y espacio (Human Development Report, 1999). A menudo, ésta se acompaña por una negligencia en el cuidado personal. La visión holística del cuidado de enfermería, el elevado número de estos profesionales y su proximidad a la población hacen de ésta, una profesión crucial para luchar contra el impacto de los efectos adversos de la globalización en la salud. Así, los enfermeros necesitan reforzar su status y aprovechar al máximo su enorme potencial (Munich Declaration: Nurses and Midwives- A force for health, 2000), y también precisan desarrollar una educación continuada en los aspectos transculturales, para afrontar los retos de la salud pública en la sociedad de nuestro tiempo. 


\section{EMIGRACIÓN}

La Conferencia Internacional sobre el Cuidado de Salud en Atención Primaria, Alma-Ata, URSS, 6-12 Septiembre 1978, en su capítulo quinto declaró: "Un objetivo social principal de los gobiernos, de las organizaciones internacionales y de las comunidades del mundo entero en las próximas décadas debe ser la consecución por todas las personas del mundo en el año 2000 de un nivel de salud que les permita dirigir una vida social y económicamente productiva". No obstante, un estudio interno, en la década de los 1990, indicó que, aunque hubo una mejoría general de la salud en Europa, no hubo ninguna progresión hacia la equidad perseguida en los años 1980. La política de intervención europea había sido mal planeada y "egoísta" en cierto modo. De nuevo, las personas con mayores necesidades eran las menos ayudadas, ya que la economía de mercado estaba actuando contrariamente a la equidad, explotando a los países con menos recursos y con una población desesperadamente necesitada.

Esta explotación también tiene lugar en los países más desarrollados, donde los inmigrantes son los más afectados, debido a su status socioeconómico menos favorecido. Pues las pobres condiciones de vida y de trabajo aumentan las diferencias entre las diferentes clases sociales. Este bajo privilegio ha sido relacionado por Bollini (1995) con el reducido acceso a los servicios de salud. De este modo, apoya la promoción de los derechos de los ciudadanos para aumentar su salud. A esto, Coote (1992) añadió que la legislación que lucha contra las desigualdades debe, asimismo, acompañarse por un esfuerzo general de la sociedad para integrar a estos grupos.

De acuerdo con Kemp (1993), estos grupos sufren diferentes problemas de salud en las comunidades que los acogen debido a sus aspectos culturales. En términos generales, se puede decir que están sometidos a conflictos psicosociales e, incluso, espirituales. Su salud se encuentra seriamente comprometida porque, mayoritariamente, su insostenible situación socioeconómica no les permite considerar la salud como una prioridad. Además de que en caso de necesidad, a menudo, experimentan un difícil acceso a los servicios del cuidado de la salud.
Las sociedades de acogida deberían ser conscientes de la etnohistoria y de la situación política de los inmigrantes, promoviendo la sensibilidad, el respeto y la empatía, si pretenden alcanzar un desarrollo sostenido. Del mismo modo, los trabajadores sociales, especialmente los enfermeros de Atención Primaria, necesitan adquirir algunos conocimientos sobre los sistemas de apoyo social, las leyes nacionales y la sociología, con el fin de ayudar a estos grupos étnicos minoritarios. La práctica enfermera precisa respaldar sus valoraciones por los aspectos culturales antes de planear las intervenciones de cuidado, con el objetivo de desarrollar habilidades clínicas competentes.

\section{DESIGUALDADES EN SALUD.}

Actualmente, la globalización está extendiéndose alrededor del mundo, y el número de inmigrantes en los países desarrollados se está haciendo cada vez más notorio. El aumento del número de grupos étnicos minoritarios está reflejándose en muchos aspectos generales de la sociedad. La cultura, la economía y la política son algunos de los más relevantes. Sin embargo, la salud es la base para desarrollar satisfactoriamente todos y cada uno de los restantes aspectos.

Desgraciadamente, también existen diferencias notables en los niveles de salud en la sociedad. Hasta ahora, ha habido muchos intentos para tratar la explicación de este fenómeno. Así, los aspectos culturales, la educación, la baja economía y la barrera del idioma han sido los más destacados (Trip-Reimer, 1985; y Gerrish, 1999), entre los factores que reducen el acceso a los servicios de salud, y que afectan directamente a las condiciones de salud.

En oposición a los aspectos culturales previos, Jenkins (1996), como resultado de su estudio, explicó que los aspectos culturales no eran decisivos en su relación con el reducido acceso a los servicios de salud. En cambio, la pobreza y el estado marital formaban las razones más consistentes.

El nivel económico es, con seguridad, un factor determinante en ambos extremos de las clases sociales. En sendas situaciones, no importa qué aspectos culturales están englobados. Muy pocos inmigrantes adinerados se encuentran en un estado poco saludable, ya que disponen de recursos para 
sobrepasar estos factores adversos. Mientras que las personas de bajo status económico, independientemente de su cultura, no son tan saludables, y experimentan, con frecuencia, dificultades en el acceso a los servicios de salud. Por otra parte, la mayoría de las personas no casadas y que, por tanto, no comparten responsabilidades, pueden practicar conductas de riesgo en su vida social y en la salud.

Las clases sociales bajas y medias también experimentan desigualdades en su salud, de acuerdo con sus diferencias étnicas. Muchos estudios han sido realizados, procurando reducir las barreras de salud, y sus resultados han fortalecido esta hipótesis. Pues sólo el empleo de trabajadores bilingües aumentó la calidad de los servicios de salud (citado por Cornwall \& Gordon, 1984), el acceso a los servicios sociales (Mori, 1994) y los resultados clínicos (Rutter, 1978).

\section{RACISMO EN LOS SERVICIOS DE SALUD Y EN LA COMUNIDAD. IDEOLOGÍAS DE AFRONTAMIENTO}

Florence Nightingale (citado por Seymer, 1954) escribió, "La mujer que enseña en India debe conocer el idioma, la religión, las supersticiones y costumbres de la mujer a la que enseña". Así, "la dama del candil" identificó por primera vez la necesidad de un cuidado cultural de alta calidad desde la profesión de enfermería.

Hoy en día, después de más de cien años, aún se documentan el cuidado inadecuado, e incluso el racismo en los servicios de salud. Por una parte, Euranis Neile (1995) detectó que los servicios de salud no cubrían las necesidades específicas de los grupos étnicos minoritarios, y que, a veces, estas necesidades, cuando se cubrían, se hacían de una forma insensible e inhumana, como en los servicios de maternidad para la comunidad china. Por otra parte, Bowler (1993), en su estudio, encontró evidencias de estereotipos cuando ciertas matronas creían que las mujeres asiáticas hacían una montaña de un grano de arena y que "no poseían instinto maternal", o Taavela (1999), quien encontró que los trabajadores de la salud percibían a los inmigrantes como clientes difíciles porque demandaban demasiado y porque no seguían las instrucciones en sus cuidados. Ambos modos contribuyen a que los grupos minoritarios experimenten desigualdades en la calidad del cuidado prestado por los servicios de salud.

Con el fin de controlar estos problemas de salud basados en aspectos étnicos, diferentes explicaciones han intentado acercarse a ellos: la multicultural, la antirracista y una nueva, que hoy en día ha emergido, la de las desigualdades entre los derechos de los ciudadanos.

La posición multicultural argumenta que la mejoría de la salud para comunidades de minorías étnicas puede ser atendida a través de la promoción de un cuidado de la salud sensible a los aspectos culturales, con tolerancia y comprensión hacia las diferentes culturas. De acuerdo con Leininger (1995), los enfermeros necesitan incorporar conocimientos de valores culturales a sus pensamientos críticos, para así proporcionar un cuidado adecuado.

La explicación antirracista apoya la necesidad de una intervención política para combatir las desigualdades de salud a través de reformas en la estructura de las relaciones sociales y facilitando el acceso al poder a individuos procedentes de estos grupos minoritarios. Esta explicación es mediada por la historia de esclavitud, colonización y dominio de la sociedad negra por la sociedad blanca. A esto, Ahmad (1993) añadió que la posición multicultural no ayuda sino a ejercer una variante del racismo.

La explicación de desigualdades en la ciudadanía intentan culpar a las diferentes oportunidades para promover los derechos y la prosperidad entre los ciudadanos de grupos étnicos minoritarios y mayoritarios. Coote (1992) argumentó que dar poder al individuo, así como ofrecerle oportunidades en la vida, deben exigirse como derechos del ciudadano. Estas variaciones socioeconómicas serán también determinantes en el acceso a los servicios de salud y educación.

Los enfermeros, en su labor para reducir las desigualdades en el cuidado de la salud, deben considerar la posición multicultural como una base teórica para sostener la práctica, la cual ayuda a incrementar la calidad de los cuidados. No obstante, un conocimiento general de los valores culturales no es suficiente. La discriminación debe ser usada para identificar a las distintas percepciones culturales de cada persona y sus necesidades, con 
el objetivo de proveer un cuidado apropiado e individualizado. Así, la equidad en los cuidados de salud se convierte en el fin último.

\section{CONTRIBUCIONES DE LOS EMIGRANTES}

La negativa situación que envuelve a los inmigrantes en sus propios países los fuerza a desplazarse en busca de mayor prosperidad. Sin embargo, las comunidades que acogen esta inmigración los han percibido siempre como una amenaza, pensando que la gente de fuera les arrebata los puestos de trabajo locales (Stalker,1997). Además, la aceptación de pobres condiciones y largos turnos de trabajo, debido a la desesperación y a la falta de regulación por las autoridades, hace que los inmigrantes se conviertan en una difícil competencia. También, la masiva entrada de inmigrantes, fuera de control por su carácter ilegal o, de nuevo, por la carencia o mala aplicación de la legislación, aumenta el desempleo, obteniendo como resultado la pobreza, la delincuencia y la explotación sexual. Estas secuelas no permiten contemplar las contribuciones positivas de la inmigración, al mismo tiempo que potencian el prejuicio, los estereotipos y el racismo.

Los beneficios para ambas comunidades son muchos. La comunidad de procedencia obtiene considerables ventajas económicas, desde reducir el excedente del desempleo, que da como resultado una mayor oportunidad de trabajo, más prosperidad y la disminución del número de personas con baja proyección social y de delincuentes. También, el dinero que envían los emigrantes a sus países de origen ayuda a obtener una mejor educación y una más alta calidad de vida a sus familias, elevando el consumo $\mathrm{y}$, consecuentemente, el empleo y las inversiones en negocios privados. Por otra parte, los emigrantes retornados aportan dinero, experiencia y nuevas aptitudes hacia los negocios. Aunque, como se sabe, no todos son beneficios, los costos también existen. La pérdida de recursos humanos, la separación familiar y la desaparición de pequeñas comunidades son algunos de ellos. En otro sentido, las comunidades de destino de los emigrantes, entre otros beneficios, experimentan un crecimiento económico, reducen la inflación, expanden su capacidad de progreso y mejoran la provisión de fuerzas de trabajo para el desarrollo de los recursos nacionales (Stalker, 1997).

Un análisis crítico de las pérdidas en las comunidades de procedencia de los emigrantes se equilibra con creces por los beneficios, destacándose el avance en la educación. Para las comunidades de destino, las ventajas también serán múltiples, siempre que exista una buena regulación por parte de las autoridades.

Asimismo, este crecimiento económico tiene una clara influencia en la salud pública, mediante la mejora en la calidad de vida para la totalidad de la sociedad. La investigación por parte de los trabajadores sociales y de la salud, y la accesibilidad a la información permiten a la mayoría de la población contribuir al incremento de su salud, reduciendo las enfermedades de transmisión y haciendo un buen uso de los servicios de salud. Sin embargo, mientras la sociedad actual en los países europeos de destino para los emigrantes está aún desarrollando estrategias para alcanzar este propósito, nuevos problemas sociales y de salud están apareciendo. La modernización e industrialización están ensanchando las diferencias entre las clases sociales, y el encuentro de diferentes culturas está, en ocasiones, promocionando el racismo. Como consecuencia, se está realzando la necesidad de una intervención política frente a los derechos de los ciudadanos, para amortiguar las desigualdades socioeconómicas.

\section{LA COMUNICACIÓN EFECTIVA}

Conforme a Hilgard Peplau (citado por Marriner, 1999), la relación interpersonal individuo-enfermero contiene las bases para que el proceso enfermero facilite la salud de los individuos y de la comunidad, ya que los enfermeros trabajan dentro de un contexto psicodinámico, donde la comunicación es la herramienta principal.

Con seguridad, se puede afirmar que el proceso de comunicación es una importante herramienta de los trabajadores de la salud para atender la salud de los individuos y de sus comunidades. Respecto a esto, se ha documentado (León Rubio, 1997) que entre el 30 y $50 \%$ de la población no sigue el tratamiento médico, debido a una comunicación poco efectiva que ocasiona como resultados, entre otros, la insatisfacción y la automedicación. 
En ocasiones, los problemas en la comunicación atienden a las diferencias, donde la comprensión, expresión, costumbres, valores y expectaciones son diferentes. Así, la comunicación efectiva puede relacionarse con la sensibilidad cultural (Andrews, 1999). En este sentido, la cultura aparece como una barrera para la comunicación efectiva, y promueve la falta de entendimiento. De esa forma, el contacto puede ser interpretado como muestra de cariño o como invasión del espacio personal (Giger, 1999).

El proceso de comunicación ha sido estudiado por muchos autores. Entre ellos, Kolb (1981) y Jenkins (1996) están de acuerdo en afirmar que las personas permanecen comunicándose todo el tiempo. Así, la emisión y recepción de información tienen lugar durante cualquier tipo de interacción, ya que la comunicación verbal se encuentra rodeada por múltiples formas de comunicación no verbal. Por ello, todas las formas de comunicación deben perseguir el mismo objetivo, de lo contrario, el resultado será diferente del buscado.

Una reciente investigación, realizada por Turner (2000), analizó los distintos aspectos que influyen en la comunicación con un objetivo predeterminado. Seguidamente, discutió la estrecha relación que poseía este tipo de comunicación con la vida social diaria, donde las experiencias previas y la comprensión son mucho más importantes que dar una información muy exacta. En este contexto, la sensibilidad cultural se somete a la participación común, donde las personas aprenden a interaccionar entre sí, creando una comunicación efectiva. Por tanto, el objetivo común entre los participantes de la comunicación se convierte en una pieza fundamental.

Sin duda, los enfermeros necesitan desarrollar nuevas habilidades en su comunicación, para así poder conseguir una buena relación desde el primer contacto con los individuos, sin que tenga importancia el grupo étnico al que pertenecen. Las respuestas efectivas durante las interacciones diarias traen comprensión, la cual es la base para una participación común. Así, tanto los enfermeros como los individuos aprenden a comunicarse efectivamente, eligiendo objetivos en el contexto de la salud, sin dar lugar a la confusión ni a malentendidos. Los individuos difícilmente restablecerán completamente su salud sin su propia colaboración, que sigue a la información y a la comunicación efectiva provista por los trabajadores de la salud.

\section{TEORÍAS DE ENFERMERÍA TRANSCULTURAL}

Según la definición de teoría de MarrinerTomey (1999), podría interpretarse que la teoría de enfermería provee de conocimientos para perfeccionar la práctica diaria de la disciplina enfermera, a través de la descripción, explicación, predicción y control del fenómeno de salud-enfermedad. Hoy en día, debido a la rápida circulación de personas alrededor del mundo, en las sociedades desarrolladas existe una mayor diversidad multicultural. Los aspectos principales de las sociedades, como valores y pensamientos, se están mezclando, y el imperialismo multicultural está tomando lugar debido al poder económico. No obstante, hay todavía un creciente número de encuentros entre las diferentes culturas. Éstos, frecuentemente, se hacen decisivos en términos de salud, por tanto, los aspectos culturales de los individuos y de sus comunidades son básicos para adecuar la valoración e intervención.

Como consecuencia, en los últimos años, varias teorías de enfermería transcultural han ido apareciendo, y todas ellas intentan adaptar el cuidado partiendo de los aspectos culturales. Entre ellas, la teoría de Madeleine Leininger ha sido la más destacada. Esta teoría hace una síntesis, por primera vez, entre la enfermería y la antropología, considerándolas mutuamente complementarias para el cuidado. Así, la práctica tradicional de cada individuo para tratar su salud, y la práctica de la enfermería deben converger en un cuidado de salud culturalmente congruente (Leininger, 1995). La valoración debe enfocarse dentro de la cultura, incluyendo las diferentes contingencias sociales y culturales, así como la etnohistoria y las creencias de salud. La intervención se divide en tres categorías: acomodación, preservación y repatronización cultural del cuidado. Estas dos últimas pueden sugerir cuestiones éticas. Así, la repatronización cuestionaría las razones de los enfermeros para crear los mayores cambios posibles en la cultura del individuo o en la comunidad; y la preservación cuestionaría la negación de la provisión de un cuidado de enfermería 
que apuntara a la más alta calidad de salud. Por tanto, la acomodación cultural del cuidado parece ser la más adecuada, al intentar encontrar un punto medio, que entre ambas culturas ejerza un efecto satisfactorio frente a la salud. La teoría transcultural de Leininger presenta múltiples interrelaciones entre los conceptos, ya de por sí complejos. Su naturaleza holística e integral relaciona estos conceptos con la estructura social, el entorno y el lenguaje, que, a la vez, se hacen indispensables para comprender sus influencias sobre los cuidados de salud. Por tanto, su aplicación requiere cierto dominio en conocimientos transculturales. Por otra parte, se puede decir que esta teoría es útil para la práctica, la educación, y para la investigación cualitativa, en el sentido de que brinda datos empíricos (Marriner-Tomey, 1999). También, se debe mencionar que esta teoría no considera los propios pensamientos de racismo y prejuicio por parte del enfermero, por lo que no desarrolla ninguna herramienta para superarlos.

Otras teorías destacan la seguridad, la sensibilidad y la competencia cultural. La teoría centrada en la seguridad cultural (Ramsdem, 1995) enfatiza el poder del individuo, una vez que está en una posición apta para ello. Para esto, los enfermeros necesitan examinar las actitudes personales, ser flexibles en sus interacciones con las personas de diferentes etnias, y evitar culparlos por su condición. Esta teoría no especifica las herramientas de intervención, y da plena autonomía a los enfermeros, quienes necesitarían de gran experiencia en este campo para aprovechar al máximo la flexibilidad que ofrece.

La teoría basada en la sensibilidad cultural subraya la necesidad de que los enfermeros posean conocimientos culturales sobre los individuos. Esta teoría ha evolucionado desde el intento de modificar el cuidado de enfermería a favor de aquellos con un reducido acceso a los servicios sociales (Bradford, 1988) hacia adaptar la práctica enfermera a las creencias del individuo, trabajando con conocimientos basados en la cultura. De este modo, los enfermeros deben explorar las creencias del individuo, para apreciar sus puntos de vista culturales y desarrollar habilidades de comunicación efectiva. También, estos profesionales deben analizar críticamente los temas que puedan afectar a la salud, para intervenir apropiadamente y aprender desde estas situaciones culturalmente significantes (Andrews \& Boyle, 1999).

Una teoría similar es la propuesta por Campinha-Bacote (1994). En ésta, la competencia cultural es el objetivo a alcanzar, y se realizaría a través del trabajo efectivo y dentro del contexto cultural del individuo o de la comunidad. De esta forma, se deben superar cuatro fases antes de obtener competencia en el cuidado cultural. Estas fases son: conciencia, conocimiento, habilidades y encuentro cultural. La conciencia cultural sería la exploración de las diferentes creencias de los individuos y los trabajadores de la salud, que afectarían a la interacción entre las personas de distintas etnias. En este sentido, estas diferencias son vistas positivamente buscando un plan de cuidados mutuo y aceptable. El conocimiento cultural es la necesidad de conocer los modos en que la cultura influye en las actitudes sobre la salud. Las habilidades culturales son las que facilitan el logro de una comunicación efectiva como herramienta de valoración cultural. Y el encuentro cultural es el compromiso con situaciones de cuidados culturales, reforzando el aprendizaje, y restando las actitudes y conocimientos equivocados. La teoría de Campinha-Bacote parece completar y desarrollar la teoría de la sensibilidad cultural, proveyendo una guía para confrontar adecuadamente las diferencias culturales en la práctica diaria. No es muy compleja, ya que explica el proceso y las herramientas paso a paso, y además puede ser aplicable a la investigación cualitativa.

\section{DISCUSIÓN}

Las teorías de enfermería transcultural sólo pretenden facilitar el proceso de atención de enfermería para ayudar a aquellas personas que sufren desigualdades de salud debido a sus características culturales. Los enfermeros deben ofrecer el mayor estándar de cuidados a su alcance, sin importarle la etnia de los individuos.

Los aspectos éticos y de educación están englobados en el objetivo "Salud para todos en el año 2000" (Conferencia de Alma-Ata, 1978). Para lograr este objetivo, los profesionales de enfermería necesitarán moverse dentro de una estructura ética, luchando contra el prejuicio, el racismo y las desigualdades en salud, con el fin de proveer cuidados 
de salud individualizados, en favor de la equidad en cuidados de salud. De igual forma, los enfermeros también precisaran de una cierta educación transcultural, ya que cuidar sin comprender los efectos de la cultura en la salud de los individuos puede dar como resultado limitar las intervenciones, que unas veces serán inútiles y otras arriesgadas. Según Abdullah (1995), los objetivos de la educación son el aumento del conocimiento transcultural, de la sensibilidad, del compromiso y de la conciencia de los diferentes valores influyentes y de las habilidades de comunicación efectiva. También, será fundamental el tiempo y la dedicación para elevar la calidad de la práctica enfermera.

\section{BIBLIOGRAFÍA}

ABDULLAH, 1995. Towards an individualized client's care: implication for education. The transcultural approach. Journal of advanced nursing. 22: 715-720.

AHMAD, W. I. U. 1993. Race in Heath in Contemporary Britain. Open University Press: Buckingham.

ANDRews, M. \& BoYLE, J. 1999. Transcultural Concepts in Nursing Care. 3rd edition. Lippincott: Philadelphia.

Anthias, F. 1988. Conference Proceedings, In Charalambous, J. Haiifanis, G., Kilonis, L. The Cypriot Community in UK: Issues of Identity. PNL Press: London.

Barnarad, A \& Spencer, J. 1996. Encyclopaedia of social and cultural anthropology. Routledge: London.

Bollini, P. \& Siem, H. 1995. No real progress towards equity: health of immigrants and ethnic minorities on the eve of the year 2000. Social Science and Medicine. 41 (6): 819-828.

Bowler, I. 1993. They're not the same as us: midwives'stereotypes of South Asian descent maternity patients. Sociology of Health and Illness. 15(2).

BRADFORD, S. 1988. Bridging the gap. Anthropological brokerage in nursing care. Unpublished Ph D dissertation, University of South Florida.

Campinha-Bacote, J. 1994. The Process of Competence in Health Care: A Culturally Competent Model of Care. 2nd Edition. Transcultural C.A.R.E. Associates: Ohio.

Coote, A. 1992. The welfare of citizens. Developing new social rights. IPPR/Rivers Oram Press: London.

Cornwall, J \& Gordon, P. 1984. An Experiment in Advocacy: The Hackney Multi-Ethnic Women's Health Project. King's Fund Centre.
Foss, G.A. 1996. A conceptual model for studying parenting behaviours in immigrant populations. Advances in Nursing Science. 19:74-87.

GerRISH, G. 1999. Inequalities in service provision: an examination of institutional influences on the provision of district nursing care to minority ethnic communities. Journal of advanced Nursing. 30:1263-1271.

Giger, J.N. \& DaVIDHIZAR, R.E. 1999. Transcultural Nursing: Assessment and Intervention. 3rd edition. Mosby: Missouri.

Human Development RePORT.1999. Oxford University Press: Oxford.

JENKINS. 1996. Social Identity. Routledge: London.

Jenkins, C.; Le T., Mcphee, S.; Stewart, S.; Ha, N. 1996. Health care access and preventive care among Vietnamese immigrants: do traditional beliefs and practices pose barriers. Social Science \& Medicine. 43: 1049-1056.

KEMP, C. 1993. Health services for refugees in countries of second asylum. International Nursing Review. 40 (1):21-24.

Kolb, I.; Rubin M. \& MCIntyre J. M. 1981. Psicologia de las Organizaciones. Experiencias. Prentice/Hall International: Madrid.

LeIninger, M. 1995. Transcultural Nursing. Concepts, Theories, Research and Practices. 2nd Ed. McGraw Hill: New York.

León Rubio, JM; Medina Anzano, S; Cantero SÁnchez, FJ; Gómez Delgado, T; Barriga JimÉnez, S; GIL RodrígueZ, F; LoSCERTALES ABRIL, F. 1997. Habilidades de información y comunicación en la atención al usuario. Junta de Andalucía, Universidad de Sevilla.

Marriner-Tomey, A. 1999. Modelos y Teorías de

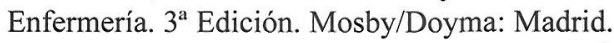

Mori, Health. 1994. Reviewuation of bilingual Health Care Schemes in East London. Mori: London.

Munich Declaration 2000. Nurses and midwifes-A force for health. http://www.who.dk/nursing

NeYLE, E. 1995. The maternity needs of the Chinese community. Nursing Times. Jan 4, 91 (1): 34-35.

RAMSDEM, I \& SPOONLEy, P. 1995. The cultural safety debate in nursing education in Aortearoa. New Zealand Annual Review of Education. 3: 161-174.

RutTer, A. C., Ross, S.E. 1978. Vancouver Perinatal Health Project: Healthiest Babies Possible: An Outreach Programme. City of Vancouver and Provincial Government of British Columbia.

Segall, H. 1990, Dasen P., Berry J., Poortinga Y. 1990. Human behaviour in global perspective. Pergamon Press: New York.

Seymer, L. 1954. Selected Writings of Florence Nightingale. McMillan: New York. 
SPECTOR, R.1991. Cultural diversity in health and illness. Appleton \& Lange. East Norwalk, CN, USA.

Stalker, P. 1997. Refugees and migration.. http://www.oneworld.org/guides/migration*

TAavela, R. (1999). The quality of immigrants' health services in primary health care in Finland. Kuopio University Publications E. Dacial Sciences. 72:196.

The International Conference on Primary Health Care, Alma-Ata, USSR, 6-12 SePTEMBER 1978. http://www.who.org.dk
Trip-Reimer, T. \& Doughert, M. 1985. Cross-cultural Nursing. Annual Review of Nursing Research. Springer Publishing Company. 3: 7-103.

TUrner, A. 2000. "Embodied ethnography: doing culture". Social Anthropology. 8 (11): 54-60.

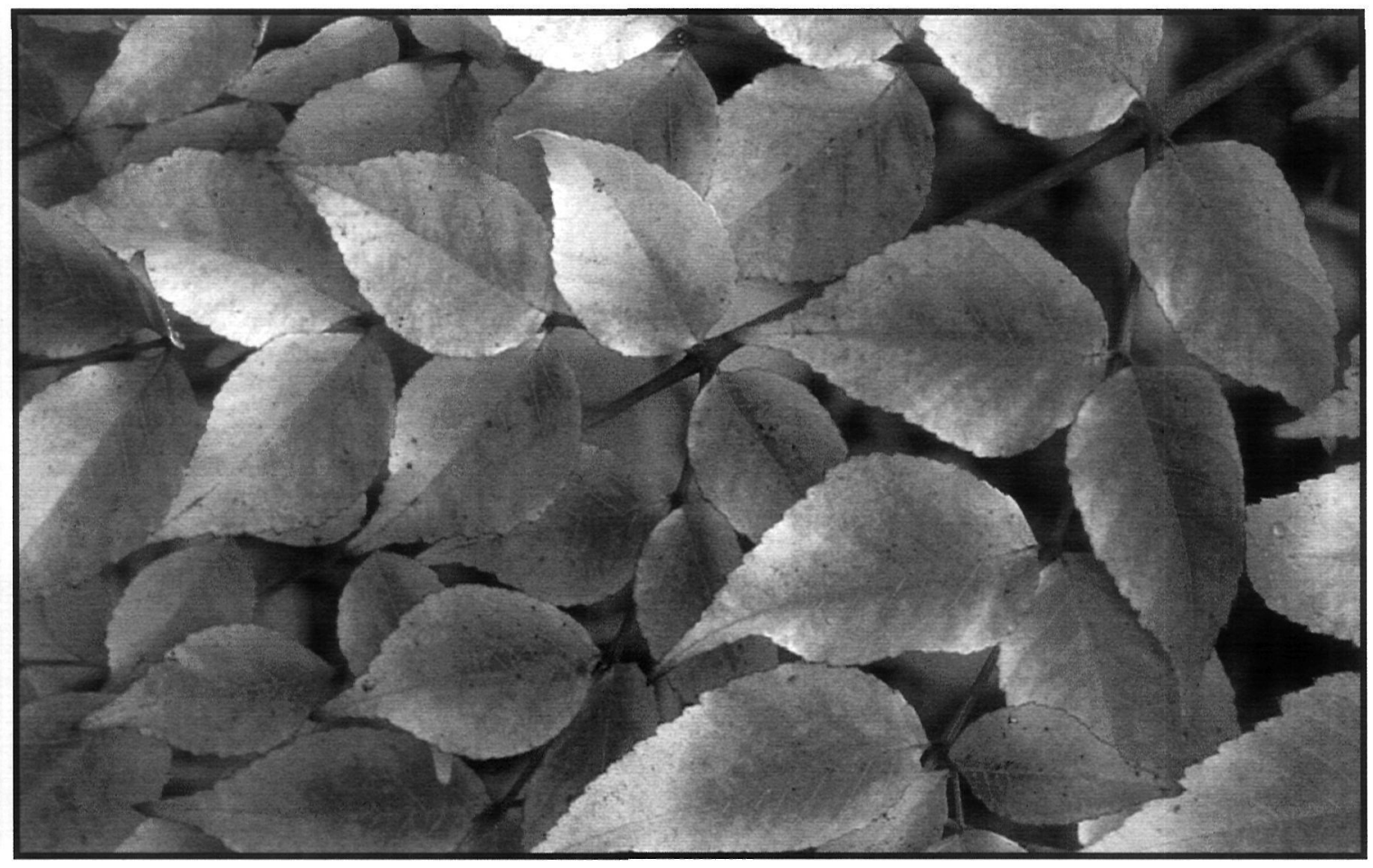

